\title{
Aplicación de redes neuronales convolucionales para la detección del tizón tardío Phytophthora infestans en papa Solanum tuberosum
}

\section{Application of convolutional neural networks for detection of the late blight Phytophthora infestans in potato Solanum tuberosum}

\author{
William Alexander Lozada-Portilla ; Marco Javier Suarez-Barón²; Eduardo Avendaño-Fernández ${ }^{3}$
}

\begin{abstract}
${ }^{1}$ Ing. Electrónico, M.Sc. Universidad Pedagógica y Tecnológica de Colombia. Sogamoso - Boyacá, Colombia; e-mail: william.lozada@uptc.edu.co; 1 https://orcid. org/0000-0003-0645-4217

${ }^{2}$ Ing. Sistemas, M.Sc, Ph.D. Universidad Pedagógica y Tecnológica de Colombia. Sogamoso - Boyacá, Colombia; e-mail: marco.suarez@uptc.edu.co; orcid.org/0000-0003-1656-4452

${ }^{3}$ Ing. Electrónico, M.Sc, Ph.D. Universidad Pedagógica y Tecnológica de Colombia. Sogamoso - Boyacá, Colombia; e-mail: eduardo.avendano@uptc.edu.co; 1 ( https://orcid.org/0000-0003-0910-8539
\end{abstract}

Cómo citar: Lozada-Portilla, W.A.; Suarez-Barón, M.J.; Avendaño-Fernández, E. 2021. Aplicación de redes neuronales convolucionales para la detección del tizón tardío Phytophthora infestans en papa Solanum tuberosum. Rev. U.D.C.A Act. \& Div. Cient. 24(2):e1917. http:// doi.org/10.31910/rudca.v24.n2.2021.1917

Artículo de acceso abierto publicado por Revista U.D.C.A Actualidad \& Divulgación Científica, bajo una Licencia Creative Commons CC BY-NC 4.0

Publicación oficial de la Universidad de Ciencias Aplicadas y Ambientales U.D.C.A, Institución de Educación Superior Acreditada de Alta Calidad por el Ministerio de Educación Nacional.

Recibido: abril 12 de 2021

Aceptado: septiembre 23 de 2021

Editado por: Helber Adrián Arévalo Maldonado

\section{RESUMEN}

La presencia del tizón tardío o gota en el cultivo de papa afecta directamente el crecimiento de la planta y el desarrollo del tubérculo, por ello, es importante la detección temprana de la enfermedad. Actualmente, la aplicación de redes neuronales convolucionales es una oportunidad orientada a la identificación de patrones en la agricultura de precisión, incluyendo el estudio del tizón tardío, en el cultivo de papa. Este estudio describe un modelo de aprendizaje profundo capaz de reconocer el tizón tardío en el cultivo de papa, por medio de la clasificación de imágenes de las hojas. Se utilizó, en la aplicación de este modelo, el conjunto de datos aumentado de PlantVillage, para entrenamiento. El modelo propuesto ha sido evaluado a partir de métricas de rendimiento, como precisión, sensibilidad, puntaje F1 y exactitud. Para verificar la efectividad del modelo en la identificación y la clasificación del tizón tardío y comparado en rendimiento con arquitecturas. como AlexNet, ZFNet, VGG16 y VGG19. Los resultados experimentales obtenidos con el conjunto de datos seleccionado mostraron que el modelo propuesto alcanza una exactitud del $90 \%$ y un puntaje F1, del $91 \%$. Por lo anterior, se concluye que el modelo propuesto es una herramienta útil para los agricultores en la identificación del tizón tardío y escalable a plataformas móviles, por la cantidad de parámetros que lo comprenden.

Palabras clave: Agricultura de precisión; Aprendizaje profundo; Redes neuronales convolucionales; Tizón tardío. 


\section{ABSTRACT}

The presence of late blight in potato crops directly affects plant growth and tuber development; therefore, early detection of the disease is important. Currently, the application of convolutional neural networks is an opportunity oriented to the identification of patterns in precision agriculture, including the study of late blight in potato crops. This study describes a deep learning model capable of recognizing late blight in potato crops by means of leaf image classification. The PlantVillage augmented dataset was used in the application of this model for training. The proposed model has been evaluated from performance metrics such as precision, sensitivity, F1 score, and accuracy; to verify the effectiveness of the model in the identification and classification of late blight and compared in performance with architectures such as AlexNet, ZFNet, VGG16, and VGG19. The experimental results obtained with the selected data set showed that the proposed model achieves an accuracy of $90 \%$ and an F1 score of $91 \%$. Therefore, it is concluded that the proposed model is a useful tool for farmers in the identification of late blight and scalable to mobile platforms due to the number of parameters that comprise it.

Keywords: Convolutional neural networks; Deep learning; Late blight; Precision agriculture.

\section{INTRODUCCIÓN}

El cultivo de papa, se destaca en Colombia como uno de los productos alimenticios de mayor impacto en las actividades agropecuarias, dado que, en torno a su explotación, se genera el desarrollo de varios sectores de la economía, como el transporte, la industria, los distribuidores de agroquímicos, la producción de empaques, entre otros (Betancourth G. et al. 2008). La papa es uno de los cultivos que mayor influencia tiene en regiones de clima frío y uno de los de mayor aumento en investigación, en todo el proceso de producción, debido a que el problema más común de este cultivo es la presencia de la gota, tizón tardío o añublo de la papa, producida por el Oomyceto Phytophthora infestans, la enfermedad más limitante, a nivel mundial, afectando, tanto a las hojas como tallos y tubérculos (Pérez \& Forbes, 2011). Los síntomas varían según el órgano afectado, la variedad y las condiciones climáticas. En hojas, el tizón tardío inicia como pequeñas manchas de color verde claro, que crecen rápidamente, tornándose de color café grisáceo.

El diagnóstico temprano de las enfermedades de las plantas es de gran importancia para la agricultura sostenible, para prevenir el desperdicio innecesario de recursos financieros, evitando usar cantidades considerables de plaguicidas y de fungicidas, para mejorar la calidad de los cultivos (Sladojevic et al. 2016). El problema en la implementación de métodos de análisis avanzados, se presenta cuando las plantas no muestran síntomas visibles en sus hojas o tallos; sin embargo, la mayoría de las enfermedades generan síntomas visibles (Atila et al. 2021). Una de las áreas de mayor investigación para mejorar las condiciones que influyen en los cultivos es la agricultura de precisión o agrónica, que incluyen algoritmos de aprendizaje de máquina (ML, por sus siglas en idioma inglés) (Sharma et al. 2021).

En los últimos años, la visión por computador, el aprendizaje automático y el aprendizaje profundo (DL, por sus siglas en idioma inglés), se han vuelto cada vez más importantes, debido a su capacidad para procesar, con alta exactitud, datos complejos (Saleem \& Chishti, 2021). Estas técnicas son las principales disciplinas para desarrollar sistemas rápidos, automáticos y precisos, para la identificación y la clasificación de imágenes (Too et al. 2019). Actualmente, se implementan sistemas que diagnostican, de forma automática, una extensa variedad de enfermedades presentes en diversos cultivos (Rangarajan Aravind et al. 2020). Para diagnosticar cuatro enfermedades, Devaraj et al. (2019) realizaron ajustes en la imagen, para posteriormente segmentarla, con la técnica de agrupación K-means y realizar la extracción de características de las hojas; dichas características fueron clasificadas por un bosque aleatorio (RF, por sus siglas en idioma inglés).

Debido a la fácil adaptación e implementación de los métodos de ML en hardware, como unidades centrales de procesamiento, unidades de procesamiento gráfico y arreglos de compuertas lógicas programables (Lecun et al. 2015), se abrieron las puertas para la implementación de nuevos métodos de alto rendimiento, lo que condujo al DL. Entre los diferentes tipos de redes neuronales profundas, las redes neuronales convolucionales ( $\mathrm{CNN}$, por sus siglas en idioma inglés) han sido las más estudiadas (Gu et al. 2018) y tienen buen rendimiento en la detección y en el diagnóstico de enfermedades, obteniendo características genéricas de los conjuntos de datos conformado por imágenes; sin embargo, existen desafíos en la implementación de CNN. Uno de ellos es recopilación de datos para el entrenamiento, puesto que se requiere un conjunto de datos bastante amplio, que contengan una gran variedad de condiciones para funcionar correctamente, además del gran esfuerzo para la recopilación (Arnal Barbedo, 2018). Por tanto, parte la comunidad científica ha optado por el uso de conjuntos de datos disponibles públicamente (Neupane \& Seok, 2020; Yin et al. 2021; Zhang et al. 2019), entre otros, como en PlantVillage (Hughes \& Salathe, 2015), el cual, es un conjunto de datos que consta de 39 clases diferentes de hojas sanas e infectadas en 14 cultivos (Xiong et al. 2020). Los conjuntos de datos actuales presentan desafíos, debido a la compleja información del fondo de las imágenes y los datos de entrenamiento, cuando son insuficientes, pueden llevar a un reconocimiento incorrecto, por lo que es necesario métodos alternativos para entrenar estos sistemas inteligentes (Geetharamani \& Arun Pandian, 2019).

Motivados por el éxito en la clasificación de imágenes por medio de CNN, Rahman et al. (2020) entrenaron arquitecturas, como VGG16, InceptionV3, MobileNet, NasNet Mobile, SqueezeNet y un modelo CNN simple; la validación utilizó un conjunto de datos que consta de 1,426 imágenes, en escenarios de la vida real, que cubren ocho enfermedades y plagas del arroz; el resultado en la identificación de las zonas afectadas obtuvo una exactitud del 93,3\%. Se evidencia que la arquitectura propuesta por los autores es 99 \% más pequeña en cantidad de parámetros que la 
comprenden, respecto a la arquitectura VGG16, lo que la hace versátil para aplicaciones móviles. Ferentinos (2018) entrenó las arquitecturas AlexNet, AlexNetOWTBn, GoogLeNet, Overfeat y VGGNet, con un conjunto de datos abierto, que consta de 87,848 imágenes, que contenían 25 plantas diferentes, con un total de 58 clases, alcanzando una tasa de éxito del $99,53 \%$ en la arquitectura VGGNet. Concluyendo que esta arquitectura se convertiría en una herramienta de advertencia o alerta temprana, muy útil para el agricultor.

Islam et al. (2019) demuestran que el aprendizaje por transferencia se puede usar para la detección temprana de enfermedades de la papa, cuando no se cuenta con un gran conjunto de datos. Los autores utilizaron 2.152 imágenes, correspondientes a dos enfermedades de la papa y hojas sanas, logrando una precisión del 99,43 \% en la arquitectura VGG16. Los autores concluyen que el resultado que obtuvieron supera a todos los trabajos existentes sobre la detección de enfermedades en la papa.

Este estudio propone una $\mathrm{CNN}$ propia, que ha sido evaluada en su rendimiento, mediante arquitecturas AlexNet, ZFNet, VGG16 y VGG19, para el diagnóstico de la enfermedad tizón tardío del cultivo de papa. Adicionalmente, se utilizaron los datos aumentados del conjunto de datos PlantVillage, obtenido de Geetharamani \& Arun Pandian (2019), el cual, se emplea en diferentes tipos de estudios para realizar análisis de comportamiento de algoritmos y modelos de IA y DL (Atila et al. 2021; Zeng et al. 2020). El principal objetivo de este estudio es analizar el rendimiento de la arquitectura de DL propuesta para la clasificación de la enfermedad tizón tardío y compararlo con otros modelos de DL.

\section{MATERIALES Y MÉTODOS}

Conjunto de datos. El conjunto PlantVillage (Hughes \& Salathe, 2015) es uno de los más utilizados en el mundo para realizar análisis de comportamiento de modelos de DL, en contextos académicos. Este conjunto de datos ocupa un tamaño de 1,67 GB y cuenta con 39 clases diferentes de hojas sanas e infectadas, que incluye alrededor de 61.486 imágenes de 14 cultivos diferentes, como manzana, mora, maíz, uva, durazno, tomate y papa. Las imágenes de este conjunto de datos, se encuentran en el modelo de color RGB, con una dimensión de 256x256 pixeles. Para este estudio, se utilizó una parte del conjunto de datos aumentado de PlantVillage, correspondiente al cultivo de papa. El fragmento del conjunto de datos utilizado consta de 3 carpetas de imágenes, correspondientes a las clases tizón temprano (1.000 imágenes), tizón tardío (1.000 imágenes) y saludable (152 imágenes), las cuales, pertenecen a muestras de cultivos de diferentes zonas del mundo. Para el caso de investigación, se utilizan las imágenes de las clases tizón tardío y saludable (Tabla 1), que competen a la enfermedad de estudio.

Tabla 1. Número de imágenes para cada clase entrenamiento/validación/ prueba.

\begin{tabular}{|c|c|c|c|c|c|c|}
\hline \multirow{2}{*}{$\begin{array}{c}\text { No. } \\
\text { clase }\end{array}$} & \multirow{2}{*}{$\begin{array}{c}\text { Nombre } \\
\text { clase }\end{array}$} & Originales & $\begin{array}{c}\text { Datos } \\
\text { aumentados }\end{array}$ & Entrenamiento & Validación & Prueba \\
\cline { 3 - 7 } & $\begin{array}{c}\text { Tizón } \\
\text { tardío }\end{array}$ & 1000 & $\begin{array}{c}1000 \\
(100 \%)\end{array}$ & $\begin{array}{c}850 \\
(85 \%)\end{array}$ & $\begin{array}{c}30 \\
(3 \%)\end{array}$ & $\begin{array}{c}120 \\
(12 \%)\end{array}$ \\
\hline 0 & Saludable & 152 & $\begin{array}{c}1000 \\
(100 \%)\end{array}$ & $(85 \%)$ & $\begin{array}{c}30 \\
(3 \%)\end{array}$ & $\begin{array}{c}120 \\
(12 \%)\end{array}$ \\
\hline
\end{tabular}

Debido a que el conjunto de datos seleccionado se encuentra desequilibrado (Tabla 1), se utiliza el conjunto de datos aumentado de PlantVillage, donde utilizaron seis técnicas de aumento diferente, para incrementar en 848 muestras adicionales, la clase saludable del conjunto de datos; las técnicas de aumento de datos utilizadas son: volteo de imágenes, corrección Gamma, inyección de ruido, aumento de color PCA, rotación y escalado (Hughes \& Salathe, 2015). El proceso del aumento de datos tiene como objetivo incrementar el número de imágenes de la clase minoritaria del conjunto de datos y ayuda a evitar el sobreajuste (overfitting) al momento de entrenar los algoritmos de ML; este problema se presenta cuando la red aprende de los casos particulares en lugar del patrón general (Peng \& Lee, 2021). Las imágenes seleccionadas, se dividieron en tres subconjuntos: entrenamiento (85\%), validación (3\%) y prueba $(12 \%)$. Se analizó el conjunto de datos, tomando un $70,75,80$ y $85 \%$, para el conjunto de datos de entrenamiento, con el fin de ir ajustado los modelos. Los modelos de CNN, se estabilizaron con una tasa del $85 \%$. En la tabla 1, se muestra el número de imágenes utilizadas en entrenamiento, en validación y en prueba. Los conjuntos de datos de entrenamiento y validación solo se utilizaron para entrenar y ajustar el modelo, mientras que el conjunto de prueba se usó para evaluar el rendimiento en el diagnóstico de muestras que el modelo no consideró antes. En el entrenamiento, se detectaron patrones de información que pueden ser utilizados en diferentes tipos de cultivos de papa que tengan la misma sintomatología y, por medio de métricas de evaluación, se estima el comportamiento de las arquitecturas.

Red neuronal convolucional. En el DL no se divide la extracción de características de la clasificación, porque la arquitectura extrae automáticamente las características, mientras se entrena; sin embargo, las CNN reducen la velocidad de entrenamiento y detección, debido al gran número de parámetros y de operaciones computacionales (Tang et al. 2020).

Arquitecturas redes neuronales convolucionales. En total cuatro arquitecturas fueron entrenadas y validadas con el conjunto de datos seleccionado: AlexNet, ZFNet y VGGNet, en sus 
versiones VGG16 y VGG19. En estas arquitecturas, se utilizó el optimizador de descenso de gradiente de código Adam, con una tasa de aprendizaje de 0,00001. La arquitectura de CNN propuesta se comparó con las arquitecturas anteriormente mencionadas.

AlexNet v1.0 es una arquitectura profunda, que contiene cinco capas convolucionales, tres totalmente conectadas y más filtros por capa, lo que mejora la extracción de características y demuestra ser una arquitectura innovadora, con los mejores resultados, hasta la fecha, en tareas de clasificación de imágenes, logrando una tasa de error del 15,3\% (Chen et al. 2021).

ZFNet v1.0 introduce mejoras significativas reduciendo el tamaño del filtro de la primera capa convolucional de 11x11 a 7x7, con pasos de 2 pixeles, en lugar de 4 . Esta nueva arquitectura retiene mucha más información en las características de la primera y segunda capa; como resultado de todas estas modificaciones, ZFNet obtuvo un error de alrededor del $16 \%$ (Fu et al. 2020).

VGGNet v1.0 VGGNet introduce un nuevo aspecto importante en el diseño de $\mathrm{CNN}$, aumentando constantemente la profundidad de la red, agregando más capas convolucionales, lo cual, es viable, debido al uso de filtros $3 \times 3$ en todas las capas, logrando un mejor resultado en la métrica de precisión en tareas de clasificación de imágenes, en dos de sus modelos: VGG16 Y VGG19. Esta arquitectura demuestra que la profundidad del mapa de características es beneficiosa para la precisión de la clasificación (Prasetyo et al. 2021).

Métricas de evaluación. Según Huerta-Mora et al. (2020), la exactitud, la sensibilidad, la especificidad, la precisión, el puntaje F1, la matriz de confusión y la curva característica operativa del receptor (ROC) son las métricas de evaluación más utilizadas y confiables para conocer el rendimiento de los modelos de CNN.

$$
\begin{aligned}
& \text { Exactitud }=\frac{T P+T N}{T P+T N+F N+F P} \\
& \text { Sensibilidad }=\frac{T P}{T P+F N} \\
& \text { Especificidad }=\frac{T N}{T N+F P} \\
& \text { Precisión }=\frac{T P}{T P+F P} \\
& \text { Puntaje F1 }=\frac{2 * T P}{2 * T P+F P+F N}
\end{aligned}
$$

Ecuación 1

Ecuación 2

Ecuación 3

Ecuación 4

Ecuación 5

Matriz de confusión: La matriz de confusión es una tabla que describe el rendimiento de los modelos de ML, se utiliza para estimar la precisión y la exactitud del modelo y es utilizada donde la salida es de dos o más tipos de clase. La matriz de confusión es un resumen de los aciertos y desaciertos realizados por la CNN en la clasificación, mediante la contabilización de cuatro variables: verdadero positivo, verdadero negativo, falso negativo y falso positivo (Huerta-Mora et al. 2020).
Curva ROC: Este gráfico evalúa la capacidad de los modelos de CNN en todos los umbrales de clasificación entre la tasa de TP y la tasa de FP, mostrando qué tan buenos son los modelos para distinguir entre las clases tizón tardío y saludable (Lobo et al. 2021).

Entrenamiento. Las arquitecturas de las CNN, se implementaron utilizando las bibliotecas pandas, numpy, tensorflow y scikit-learn, que utilizan el lenguaje de programación Python. El entrenamiento y la validación, se realizó en Google Cola, mientras que el test de las arquitecturas entrenadas, sobre un computador portátil ASUS S46C, con un Core i $73^{\mathrm{a}}$ Gen - (8GB/1TB HDD/Windows 10 Pro) y equipado con tarjeta gráfica NVIDIA GeForce GT $740 \mathrm{M}$, en el entorno de desarrollo Jupyter Notebook usando Python 3.7.

Los parámetros de entrenamiento fueron los mismos para todas las arquitecturas y se seleccionaron con base en Geetharamani \& Arun Pandian (2019), quienes realizaron variaciones en los parámetros de las $\mathrm{CNN}$ en el entrenamiento. El optimizador utilizado para el entrenamiento es el optimizador Adam, con una tasa de aprendizaje de 0,00001 (Lee et al. 2020) y la cantidad de épocas seleccionada fue 250. En este estudio, cada valor de píxel de las imágenes del conjunto de datos utilizado se normalizó dividiendo por 255 . Luego, las imágenes se redimensionaron al tamaño predeterminado aceptado por cada CNN.

\section{RESULTADOS Y DISCUSIÓN}

Con base en los resultados de las matrices de confusión de la figura 1, se hallaron las métricas de evaluación de las ecuaciones 1-5. La tabla 3 muestra la exactitud o tasa de aciertos totales de cada modelo con el conjunto de datos de prueba. La arquitectura VGG16 logró el mejor rendimiento respecto a las demás arquitecturas, alcanzando una exactitud del $96 \%$, mientras que AlexNet presentó el segundo mejor rendimiento, logrando una exactitud del $94 \%$. Por otra parte, la arquitectura ZFNet obtuvo un rendimiento ligeramente menor a las arquitecturas AlexNet y VGG16, mientras que la arquitectura VGG19 arrojó los valores de exactitud más bajos con respecto a las otras tres arquitecturas, alcanzando apenas un $79 \%$ en la exactitud. La sensibilidad o eficiencia de las arquitecturas de CNN para detectar muestras que efectivamente presentan la enfermedad tizón tardío en las hojas de papa, expone que VGG16 presentó el mejor rendimiento, con un $92 \%$ de sensibilidad, mientras que AlexNet logró el segundo mejor rendimiento, con un $88 \%$ de sensibilidad.

La especificidad o la capacidad del clasificador para detectar las muestras que efectivamente son negativas o no presentan la enfermedad tizón tardío, se muestra en la tabla 3, evidenciando que todas las arquitecturas clasifican de manera satisfactoria las hojas sanas. La precisión de las arquitecturas de CNN para clasificar hojas correctamente con la enfermedad tizón tardío, también se muestra en la tabla 3, señalando que las arquitecturas presentan un excelente desempeño al clasificar las hojas que, efectivamente, tienen la enfermedad tizón tardío, dado que ninguna arquitectura presenta algún FP en la matriz de confusión. Por último, la métrica puntaje $\mathrm{F} 1$ evidencia que, en las CNN, la precisión y la sensibilidad son igual de importantes; los resultados encontrados muestran que 


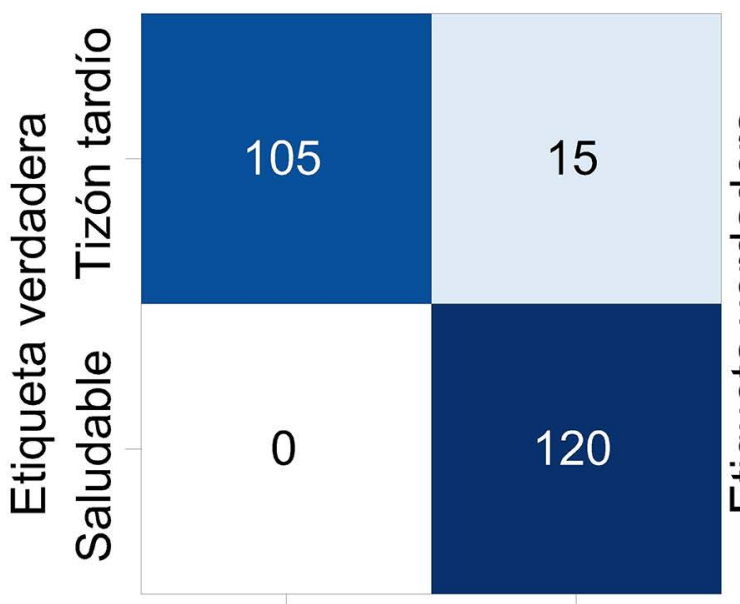

Tizón tardío Saludable Etiqueta predicha

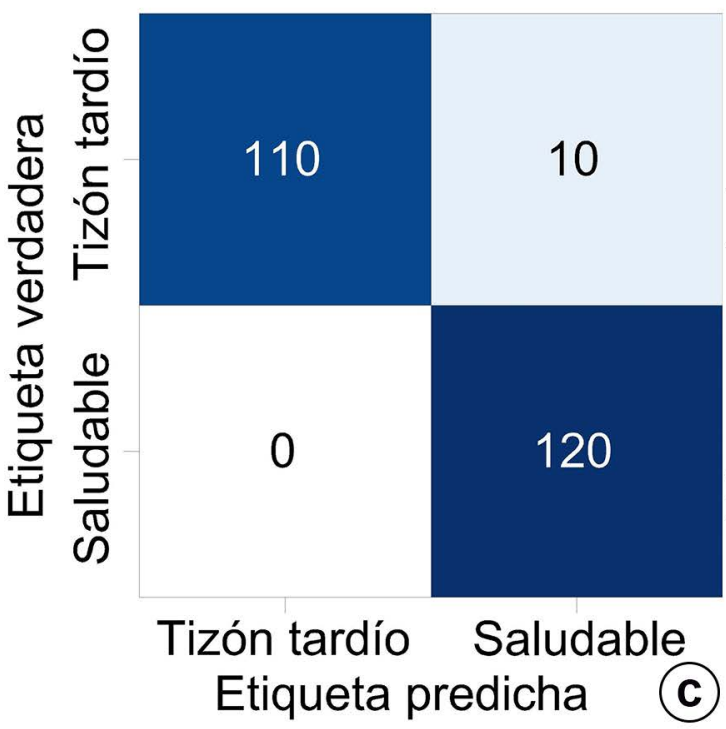

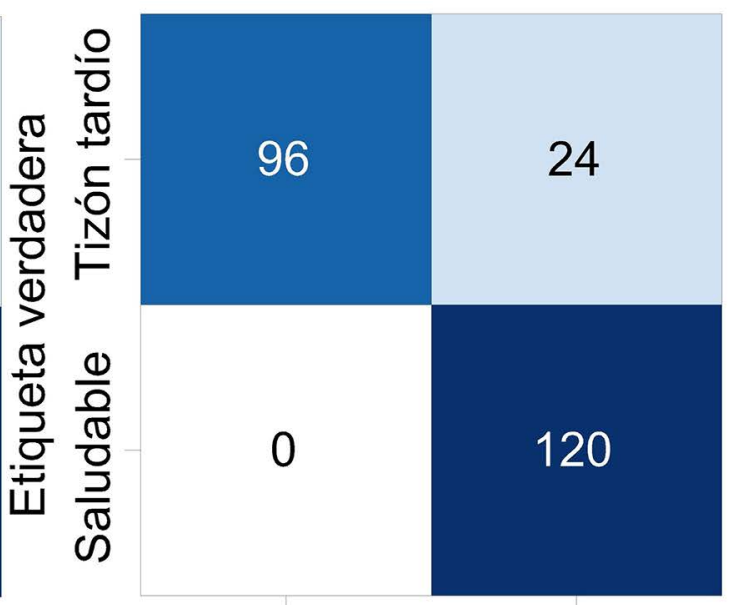

Tizón tardío Saludable Etiqueta predicha
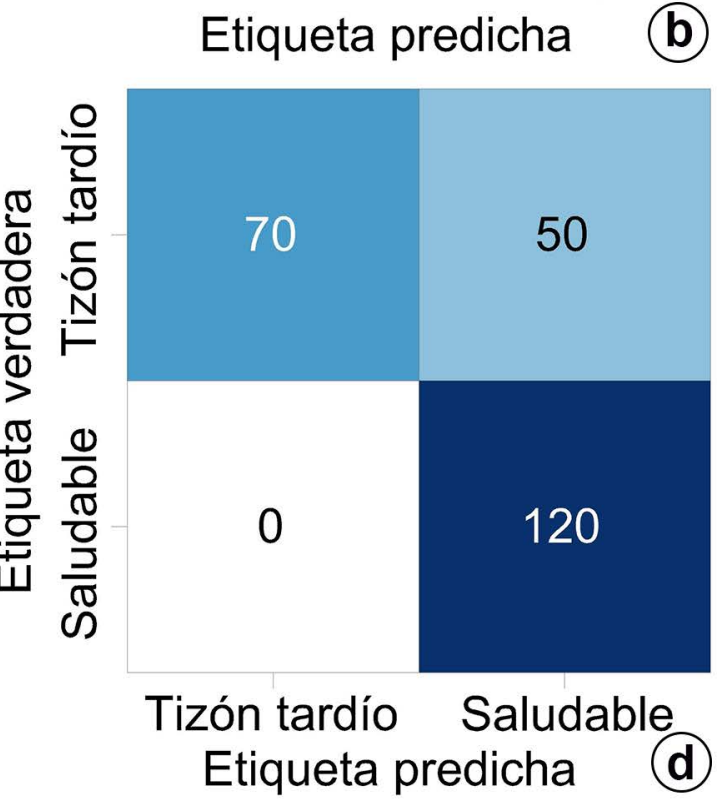

Figura 1. Matrices de confusión de las arquitecturas de CNN. a. AlexNet; b. ZFNet; c. VGG16; d. VGG19.

las dos mejores arquitecturas son VGG16 y AlexNet, logrando un puntaje F1 del 96 y del $93 \%$, respectivamente.

La figura 2 evidencia cuáles fueron arquitecturas que extrajeron los modelos genéricos del conjunto de datos y presentaron un rendimiento óptimo en todas las métricas de evaluación presentadas. La curva ROC muestra la relación entre tasa de TP y la tasa de FP, observando que las mejores arquitecturas que distinguen entre hojas sanas y hojas infectadas con tizón tardío son VGG16 y AlexNet.

Además de entrenar y evaluar los modelos CNN de última generación, se construyó desde cero una arquitectura $\mathrm{CNN}$, que consta de 11 capas, para una imagen de entrada de 227x227 pixeles (Tabla 2), inspirada en la naturaleza secuencial de VGG16. El modelo propuesto, se entrenó bajo las mismas condiciones que los otros modelos de $\mathrm{CNN}$, con el fin de comparar las arquitecturas bajo las mismas configuraciones. El rendimiento de la arquitectura propuesta, se compara con las arquitecturas que mejor rendimiento presentaron en las métricas de evaluación, para este estudio: VGG16 y AlexNet. El modelo propuesto obtuvo un rendimiento cercano a los modelos que mejores resultados presentaron en las pruebas, logrando una exactitud de $5 \%$ menos respecto a VGG16 y $3 \%$ menos respecto a AlexNet (Tabla 3). Por otra parte, la arquitectura propuesta presentó un porcentaje de entre el 6-3\% menos en el puntaje F1 respecto a las arquitecturas VGG16 y AlexNet (Tabla 3).

El puntaje $\mathrm{F} 1$, se considera la métrica de rendimiento más importante, por lo que la arquitectura con el valor mayor en esta métrica se consideró la arquitectura de mejor rendimiento en la identificación y en la clasificación del tizón tardío en el cultivo de papa, la cual, fue VGG16. Esta es una arquitectura consistente y eficiente que obtuvo una puntuación superior al $92 \%$ en todas las métricas de evaluación (Tabla 3). Otra de las arquitecturas que presentó un excelente desempeño fue AlexNet, que obtuvo un 


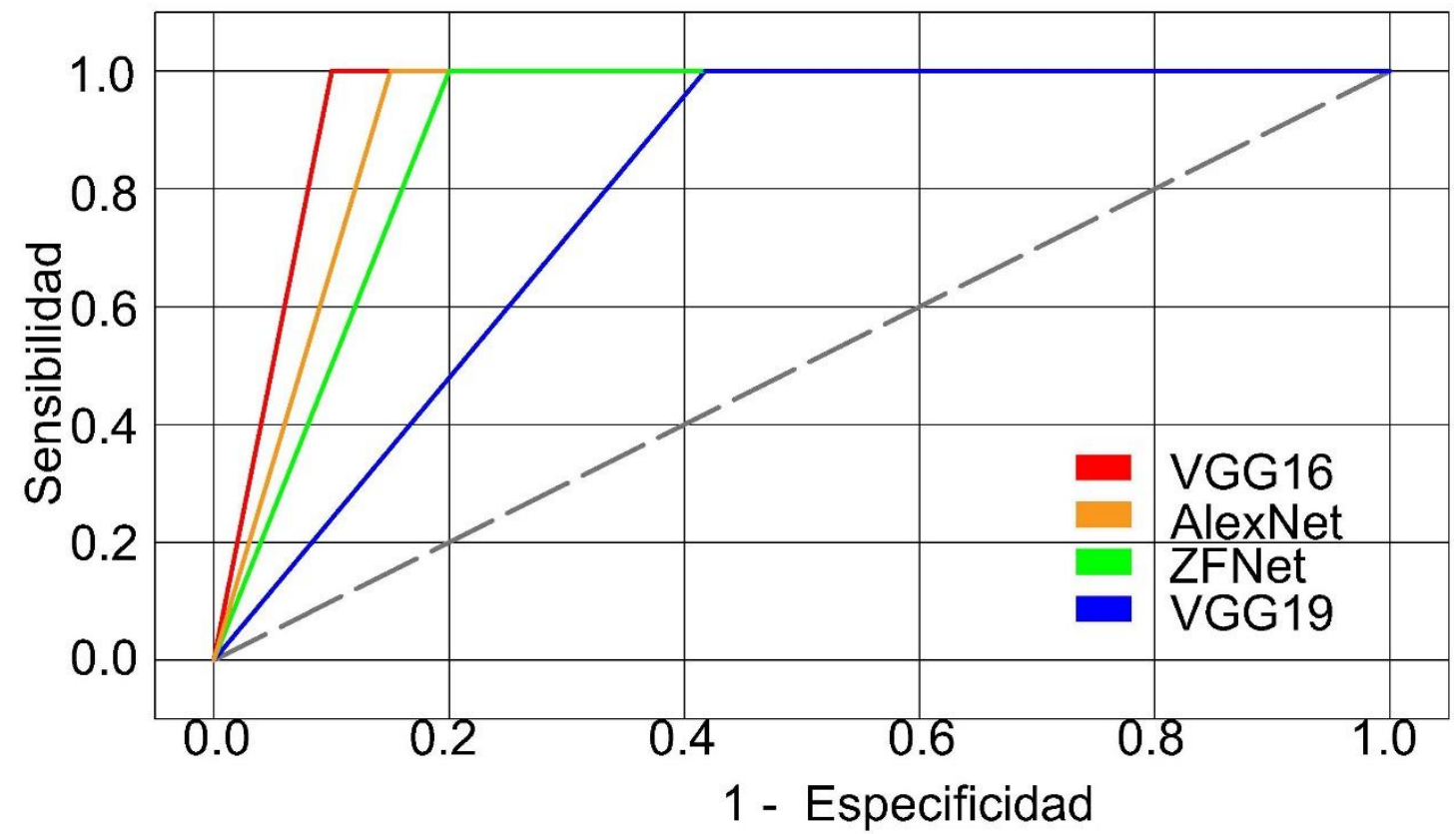

Figura 2. Curvas ROC de los modelos de CNN.

Tabla 2. Detalles del modelo propuesto.

\begin{tabular}{|l|c|c|c|c|}
\hline \multicolumn{1}{|c|}{ Capa } & $\begin{array}{c}\text { Forma de } \\
\text { salida }\end{array}$ & $\begin{array}{c}\text { Tamaño } \\
\text { Filtro/Paso }\end{array}$ & Relleno & Activación \\
\hline Imagen & $(224,224,003)$ & - & - & - \\
\hline Convolución & $(224,224,064)$ & $(3,3) / 1$ & 'same' & 'relu' \\
\hline Agrupamiento & $(112,112,064)$ & $(2,2) / 2$ & - & - \\
\hline Convolución & $(112,112,128)$ & $(3,3) / 1$ & 'same' & 'relu' \\
\hline Agrupamiento & $(112,112,128)$ & $(3,3) / 1$ & 'same' & 'relu' \\
\hline Convolución & $(56,56,128)$ & $(2,2) / 2$ & - & - \\
\hline Convolución & $(56,56,256)$ & $(3,3) / 1$ & 'same' & 'relu' \\
\hline Agrupamiento & $(56,56,256)$ & $(3,3) / 1$ & 'same' & 'relu' \\
\hline Convolución & $(56,56,256)$ & $(3,3) / 1$ & 'same' & 'relu' \\
\hline Convolución & $(28,28,256)$ & $(2,2) / 2$ & - & - \\
\hline Agrupamiento & $(28,28,512)$ & $(3,3) / 1$ & 'same' & 'relu' \\
\hline Convolución & $(28,28,512)$ & $(3,3) / 1$ & 'same' & 'relu' \\
\hline Convolución & $(28,28,512)$ & $(3,3) / 1$ & 'same' & 'relu' \\
\hline Convolución & $(14,14,512)$ & $(2,2) / 2$ & - & - \\
\hline Agrupamiento & $(14,14,512)$ & $(3,3) / 1$ & 'same' & 'relu' \\
\hline Flatten & $(25088)$ & - & - & - \\
\hline FC & $(4096)$ & - & - & 'relu' \\
\hline FC & $(4096)$ & - & - & 'softmax' \\
\hline Salida & $(2)$ & - & & \\
\hline
\end{tabular}

mejor rendimiento en función de la cantidad de parámetros que la componen. AlexNet tiene, aproximadamente, 58,3 millones de parámetros, mientras que VGG16, cerca de 134,3 millones de parámetros. Realizando una comparación directa del puntaje F1 entre estas dos arquitecturas, AlexNet alcanza $93 \%$, mientras que VGG16, el $96 \%$. Esta es una diferencia mínima en el puntaje F1, que se ve traducido en 5 clasificaciones mal realizadas entre una arquitectura y otra, lo que hace que AlexNet sea la mejor opción de las arquitecturas estudiadas.
El rendimiento de la arquitectura propuesta comparado con AlexNet, se puede considerar que es bueno y prometedor, debido a que el desempeño de la arquitectura propuesta es cercano a AlexNet y, además, es un modelo que ocupa un ligero tamaño en disco y tiene una arquitectura profunda. Esto puede hacer que nuestro modelo realice una mejor abstracción de las características genéricas si se aumenta el conjunto de datos o se aumenta el número de clases. 
Tabla 3. Valores de las métricas de evaluación de la arquitectura propuesta con las arquitecturas de mejor rendimiento.

\begin{tabular}{|l|c|c|c|c|c|}
\hline \multicolumn{1}{|c|}{ Modelo } & Exactitud & Sensibilidad & Especificidad & Precisión & $\begin{array}{c}\text { Puntaje } \\
\text { F1 }\end{array}$ \\
\hline AlexNet & 0,94 & 0,88 & 1,00 & 1,00 & 0,93 \\
\hline ZFNet & 0,90 & 0,80 & 1,00 & 1,00 & 0,89 \\
\hline VGG16 & 0,96 & 0,92 & 1,00 & 1,00 & 0,96 \\
\hline VGG19 & 0,79 & 0,58 & 1,00 & 1,00 & 0,74 \\
\hline $\begin{array}{l}\text { Arquitectura } \\
\text { propuesta }\end{array}$ & 0,91 & 0,83 & 1,00 & 1,00 & 0,90 \\
\hline
\end{tabular}

Al comparar la exactitud de la arquitectura propuesta con estudios similares encontrados en la literatura, se encuentra que Islam et al. (2019) obtienen una exactitud del 99,43\%, pero utilizan el aprendizaje por transferencia para entrenar la CNN, propuesta por los autores, empleando VGG16; por otro lado, Militante et al. (2019) logran una exactitud del $96,5 \%$, con la única diferencia que utiliza un conjunto de datos más amplio de 35.000 imágenes. Por último, en el estudio de Lee et al. (2020), se obtuvo una exactitud de $99 \%$; en este estudio, los autores realizaron un ajuste en las imágenes, eliminando el fondo y dejando solo la hoja de la papa en la imagen para el posterior entrenamiento de la CNN. Por tanto, es posible utilizar eficazmente la arquitectura propuesta de CNN para diagnóstico temprano del tizón tardío en el cultivo de papa, procesando cada imagen y proporcionando resultados altamente precisos en tiempo real, debido al tamaño de la arquitectura y a la cantidad de parámetros que la comprende. Dichas características hacen que la arquitectura propuesta sea idónea para la creación de diversos tipos de interfaces humano máquina. Como un trabajo futuro, se pretende estructurar el conjunto de datos propio, enfocado en las variedades de papa cultivadas de la sabana cundiboyacense y realizar el análisis de comportamiento de las arquitecturas de CNN en nuestro contexto.

Conflictos de intereses: El manuscrito fue preparado y revisado con la participación de todos los autores, quienes declaramos que no existe ningún conflicto de intereses que ponga en riesgo la validez de los resultados presentados.

\section{REFERENCIAS}

1. ARNAL BARBEDO, J.G. 2018. Impact of dataset size and variety on the effectiveness of deep learning and transfer learning for plant disease classification. Computers and Electronics in Agriculture. 153:46-53. https://doi.org/10.1016/j.compag.2018.08.013

2. ATILA, U.; UCAR, M.; AKYOL, K.; UCAR, E. 2021. Plant leaf disease classification using efficientNet deep learning model. Ecological Informatics. 61(2021):101182. https://doi.org/10.1016/j.ecoinf.2020.101182

3. BETANCOURTH G., C.; PORTILlA B., E.; SALAS P., H. 2008. Evaluación de la reacción de nueve genotipos de papa (Solanum tuberosum Subsp. Andigena) al ataque de Phytophthora infestans (Mont) de Bary. Agronomía Colombiana (Colombia). 26(3):411-116.

4. CHEN, J.; WAN, Z.; ZHANG, J.; LI, W.; CHEN, Y.; LI, Y.; DUAN, Y. 2021. Medical image segmentation and reconstruction of prostate tumor based on 3D AlexNet. Computer Methods and Programs in Biomedicine. 200:105878. https://doi.org/10.1016/j.cmpb.2020.105878

5. DEVARAJ, A.; KARUNYA, R.; JAAHNAVI, S.; INDIRA, K. 2019. Identification of plant disease using image processing technique. Proceedings of the 2019 IEEE International Conference on Communication and Signal Processing, ICCSP. p.749-753.

https://doi.org/10.1109/ICCSP.2019.8698056

6. FERENTINOS, K.P. 2018. Deep learning models for plant disease detection and diagnosis. Computers and Electronics in Agriculture. 145(2018):311-318.

https://doi.org/10.1016/j.compag.2018.01.009

7. FU, L.; MAJEED, Y.; ZHANG, X.; KARKEE, M.; ZHANG, Q. 2020. Faster R-CNN-based apple detection in densefoliage fruiting-wall trees using RGB and depth features for robotic harvesting. Biosystems Engineering. 197:245-256. https://doi.org/10.1016/j.biosystemseng.2020.07.007

8. GEETHARAMANI, G.; ARUN PANDIAN, J. 2019. Identification of plant leaf diseases using a nine-layer deep convolutional neural network. Computers and Electrical Engineering. 76(2019):323-338.

https://doi.org/10.1016/j.compeleceng.2019.04.011

9. GU, J.; WANG, Z.; KUEN, J.; MA, L.; SHAHROUDY, A.; SHUAI, B.; LIU, T.; WANG, X.; WANG, G.; CAI, J.; CHEN, T. 2018. Recent advances in convolutional neural networks. Pattern Recognition. 77(2018):354-377. https://doi.org/10.1016/j.patcog.2017.10.013

10. HUERTA-MORA, E.A.; GONZÁLEZ-HUITRÓN, V.; RODRÍGUEZ-RANGEL，H.; AMABILIS-SOSA, L.E. 2020. Detección de enfermedades foliares con arquitecturas de redes neuronales convolucionales. Revista Internacional De Desarrollo Regional Sustentable. 5(1):18-40. 
11. HUGHES, D.P.; SALATHE, M. 2015. An open access repository of images on plant health to enable the development of mobile disease diagnostics. (Suiza) arXiv e-prints. Disponible desde Internet en:

http://arxiv.org/abs/1511.08060 (con acceso el 4/12/2021)

12. ISLAM, F; HOQ, M.N.; RAHMAN, C.M. 2019. Application of transfer learning to detect potato disease from leaf image. 2019 IEEE International Conference on Robotics, Automation, Artificial-Intelligence and Internet-of-Things (RAAICON). p.127-130.

https://doi.org/10.1109/RAAICON48939.2019.53

13. LECUN, Y.; BENGIO, Y.; HINTON, G. 2015. Deep learning. Nature (USA). 521(7553):436-444.

https://doi.org/10.1038/nature14539

14. LEE, T.Y.; YU, J.Y.; CHANG, Y.C.; YANG, J.M. 2020. Health Detection for Potato Leaf with Convolutional Neural Network. Indo - Taiwan 2nd International Conference on Computing, Analytics and Networks, Indo-Taiwan ICAN 2020 - Proceedings. p.289-293.

https://doi.org/10.1109/Indo-TaiwanICAN48429.2020.9181312

15. LOBO, G.P.; LARAWAY, J.; GADGIL, A.J. 2021. Identifying schools at high-risk for elevated lead in drinking water using only publicly available data. Science of The Total Environment. 803:150046.

https://doi.org/10.1016/j.scitotenv.2021.150046

16. MILITANTE, S.V.; GERARDO, B.D.; DIONISIO, N.V. 2019. Plant Leaf Detection and Disease Recognition Using Deep Learning. (Taiwan) 2019 IEEE Eurasia Conference on IOT, Communication and Engineering, ECICE 2019. p.579-582. https://doi.org/10.1109/ECICE47484.2019.8942686

17. NEUPANE, D.; SEOK, J. 2020. Bearing fault detection and diagnosis using case western reserve university dataset with deep learning approaches: A review. IEEE Access. 8:93155-93178.

https://doi.org/10.1109/ACCESS.2020.2990528

18. PENG, Y.L.; LEE, W.P. 2021. Data selection to avoid overfitting for foreign exchange intraday trading with machine learning. Applied Soft Computing. 108:107461. https://doi.org/10.1016/j.asoc.2021.107461

19. PÉREZ, W.; FORBES, G. 2011. Guía de identificación de plagas que afectan a la papa en la zona andina. Centro Internacional de la Papa -CIP. p.44.

20. PRASETYO, E.; SUCIATI, N.; FATICHAH, C. 2021. Multi-level residual network VGGNet for fish species classification. Journal of King Saud University - Computer and Information Sciences.

https://doi.org/10.1016/j.jksuci.2021.05.015
21. RAHMAN, C.R.; ARKO, P.S.; ALI, M.E.; IQBAL KHAN, M.A.; APON, S.H.; NOWRIN, F.; WASIF, A. 2020. Identification and recognition of rice diseases and pests using convolutional neural networks. Biosystems Engineering. 194(2020):112-120. https://doi.org/10.1016/j.biosystemseng.2020.03.020

22. RANGARAJAN ARAVIND, K.; MAHESWARI, P.; RAJA, P.; SZCZEPAŃSKI, C. 2020. Crop disease classification using deep learning approach: an overview and a case study. In: Das, H.; Pradhan, C.; Dey, N. Deep Learning for Data Analytics. Academic Press. Polonia. p.173-195. https://doi.org/10.1016/b978-0-12-819764-6.00010-7

23. SALEEM, T.J.; CHISHTI, M.A. 2021. Deep learning for the internet of things: Potential benefits and use-cases. Digital Communications and Networks. https://doi.org/10.1016/j.dcan.2020.12.002

24. SHARMA, A.; JAIN, A.; GUPTA, P.; CHOWDARY, V. 2021. Machine Learning Applications for Precision Agriculture: A Comprehensive Review. IEEE Access. 9:4843-4873. https://doi.org/10.1109/ACCESS.2020.3048415

25. SLADOJEVIC, S.; ARSENOVIC, M.; ANDERLA, A.; CULIBRK, D.; STEFANOVIC, D. 2016. Deep neural networks based recognition of plant diseases by leaf image classification. Computational Intelligence and Neuroscience. 2016:11 https://doi.org/10.1155/2016/3289801

26. TANG, Z.; YANG, J.; LI, Z.; QI, F. 2020. Grape disease image classification based on lightweight convolution neural networks and channelwise attention. Computers and Electronics in Agriculture. 178:105735. https://doi.org/10.1016/j.compag.2020.105735

27. TOO, E.C.; YUJIAN, L.; NJUKI, S.; YINGCHUN, L. 2019. A comparative study of fine-tuning deep learning models for plant disease identification. Computers and Electronics in Agriculture. 161:272-279.

https://doi.org/10.1016/j.compag.2018.03.032

28. XIONG, Y.; LIANG, L.; WANG, L.; SHE, J.; WU, M. 2020. Identification of cash crop diseases using automatic image segmentation algorithm and deep learning with expanded dataset. Computers and Electronics in Agriculture. 177:105712 https://doi.org/10.1016/j.compag.2020.105712

29. YIN, W.; SUN, X.; DIAO, W.; ZHANG, Y.; GAO, X. 2021. Thermal power plant detection in remote sensing images with saliency enhanced feature representation. IEEE Access. 9:8249-8260. https://doi.org/10.1109/ACCESS.2021.3049431 
30. ZENG, Q.; MA, X.; CHENG, B.; ZHOU, E.; PANG, W. 2020. GANS-based data augmentation for citrus disease severity detection using deep learning. IEEE Access. 8:172882-172891.

https://doi.org/10.1109/ACCESS.2020.3025196
31. ZHANG, Z.; GAO, W.; ZHANG, F.; HUANG, Y.; DAI, S.; FAN, F.; ZHAN, J.; DU, M.; YIN, S.; XIONG, L.; DU, J.; CHENG, Y.; ZHOU, X.; REN, R.; WANG, L.; YE, H. 2019. Landscape of big medical data: a pragmatic survey on prioritized tasks. IEEE Access. 7:15590-15611. https://doi.org/10.1109/ACCESS.2019.2891948 